\title{
DER EINFLUSS VON CHLORCHOLINCHLORID (CCC) AUF WACHSTUM, ERTRAG UND NÄHRSTOFFAUFNAHME VON SOMMERWEIZEN IN EINEM FELDVERSUCH
}

\author{
Antti JaAkKola \\ Universität Helsinki, Institut für Agrikulturchemie
}

Eingegangen am 20. 2. 1967

Versuche zur Ermittlung der Verwendung des Chlorcholinchlorids (CCC) zur Erhöhung der Standfestigkeit des Sommer- oder Winterweizens haben seit 1960 stattgefunden, als "Die Biologische Forschungsabteilung der österreichischen Stickstoffwerke AG» ihren ersten Gefässversuch mit wechselnden Mengen des CCC durchführte. Aufgrund der Ergebnisse dieses Versuches begann das Institut im Jahre 1961 eine Feldversuchsserie mit Winterweizen (MAYR, Primost und RitTMeYer 1962, PRIMOsT 1964). In diesen Versuchen wurde das CCC anfangs wie ein Düngemittel, später teils in gelöster Form verabreicht.

Für die Verwendung des CCC als Spritzmittel sprechen die Ergebnisse von zwanzig in den Jahren 1962-63 in Deutschland durchgeführten Feldversuchen (STURM und Jung 1964). In allen Feldversuchen in Finnland ist das CCC auf junge Pflanzen gespritzt worden (Mukula, Teittinen und LaAksonheimo 1965).

In beinahe allen mit Weizen durchgeführten CCC-Versuchen ist eine deutliche Halmverkürzung hervorgerufen worden, was in den meisten Fällen zu einer Erhöhung der Standfestigkeit geführt hat. Von dem Einfluss des CCC auf den Ertrag und auf die Nährstoffzusammensetzung des Ertrages hat man beträchtlich voneinander abweichende Ergebnisse. Im allgemeinen hat sich der Nährstoffgehalt des Ertrages erhöht, indem sich der Ertrag verringert hat.

Um die Wirkungen des auf junge Pflanzen der Sommerweizensorte Norröna gespritzten CCC in den Verhältnissen Südfinnlands zu erforschen, wurde in Hausjärvi $\left(60^{\circ} 46^{\prime}\right.$ nördl. Breite, $24^{\circ} 51^{\prime}$ östl. Länge) ein Feldversuch durchgeführt, über den in diesem Aufsatz berichtet wird. 


\section{Versuchsmethodik}

Der Boden des Versuchsfeldes war lehmiger Sand. Der pH-Wert in 0,01-m $\mathrm{CaCl}_{2}$ war in dem Oberboden $\mathrm{pH} \mathrm{6,55}$ und im Unterboden $\mathrm{pH} 5,6$. Der Oberboden war ziemlich humusreich $(3.75 \%$ org. C) und sein Stickstoffzustand mässig $(2.18 \%$ tot. $\mathrm{N}$ und $52 \mathrm{mg} / \mathrm{kg}$ nachlieferbaren $\mathrm{NO}_{3}-\mathrm{N}$ ). Der Kalivorrat des Bodens war gross, weswegen die zum Versuch als Versuchsfaktor gehörende Kalidüngung bei der Betrachtung der Ergebnisse unberücksichtigt gelassen werden konnte. Ein zureichender Vorrat an Phosphor wurde mit $500 \mathrm{~kg}$ Superphosphat/ha besorgt.

Der Versuch wurde als Polyfaktorversuch mit der Teilparzellenmethode ausgeführt. $\mathrm{Zu}$ den Teilparzellen gehörten die Stufen der CCC-Spritzung $\left(\mathrm{c}_{\mathbf{0}}=\right.$ unbehandelt, $\mathrm{c}_{1}=$ gespritzt mit $\left.3 \mathrm{~kg} \mathrm{CCC} / \mathrm{ha}\right)$, zu den Hauptparzellen die drei Stufen der Stickstoffdüngung $\left(\mathrm{n}_{1}=25 \mathrm{~kg} \mathrm{~N} / \mathrm{ha}, \mathrm{n}_{2}=50 \mathrm{~kg} \mathrm{~N} / \mathrm{ha}, \mathrm{n}_{3}=100 \mathrm{~kg} \mathrm{~N} / \mathrm{ha}\right)$. Als Stickstoffdüngemittel wurde Kalkammonsalpeter $\left(12.5 \% \mathrm{NH}_{4}-\mathrm{N}, 12.5 \% \mathrm{NO}_{3}-\mathrm{N}\right)$ verwendet. $\mathrm{Zu}$ dem Versuch gehörten vier vollständige Blocks. Die Grösse der Teilparzelle war $2.05 \times 28 \mathrm{~m}$, zu einer Hauptparzelle gehörten zwei nebeneinander liegende Teilparzellen.

Der Versuch wurde am 15. 5. besät, wovor sowohl die Grunddüngung als die Stickstoffdüngung ausgeführt worden waren. Das Feld spross am 26. 5. auf. Die CCC-Spritzung der $c_{1}$-Parzellen wurde am 15. 6. ausgeführt, als die Pflanzen das 4-Blatt-Stadium und eine Höhe von $20 \mathrm{~cm}$ hatten. Pflanzenproben wurden am 27. 6., 21. 7. und 15. 8. gesammelt. Am 4. 8. wurde die Standfestigkeit geschätzt. Am 8. 9. wurden die Ährenproben gesammelt, um die Unterschiede im Reifen zu untersuchen. Der Versuch wurde am 18. 9. mit Mähdrescher abgeerntet, als das Korn vollreif war. Die wegen der Entwicklung der Versuchspflanze wichtigen Zeitpunkte werden in der Abbildung 1 angeführt, in der die klimatischen Verhältnisse der Versuchszeit mit den Temperaturen (um $20 \mathrm{Uhr}$, als Bruchstrich) und Niederschlägen (als Säulen) beschrieben werden.

\section{Ergebnisse}

Wa chst u m und En t w i c k l ung. Der Einfluss des CCC auf das Längenwachstum der Pflanze war schon zwölf Tage nach der Spritzung deutlich, was aus den unten stehenden Ziffern hervorgeht, mit denen die Länge der am 27.6. genommenen Pflanzenproben von der Bodenoberfläche bis zur Spitze des längsten Blattes in Zentimeter angeführt wird. Die Ergebnisse sind Mittelwerte von 240 Messungen.

$\begin{array}{ccccc} & \mathrm{n}_{1} & \mathrm{n}_{2} & \mathrm{n}_{3} & \overline{\mathrm{n}} \\ \mathrm{c}_{0} & 32.7 & 35.9 & 37.7 & 35.4 \\ \mathrm{c}_{1} & 29.4 & 32.2 & 34.4 & 32.0 \\ \overline{\mathrm{c}} & 31.1 & 34.1 & 36.0 & 33.7\end{array}$

Die CCC-Spritzung hatte also zu dieser Zeit eine um $3.4 \mathrm{~cm} * *$ verkürzende Einwirkung $\left(\mathrm{C}=\overline{\mathrm{c}}_{1}-\overline{\mathrm{c}}_{\mathbf{0}}\right)$ auf die Pflanzen. Die lineare Wirkung der Stickstoffdüngung $\left(\mathrm{N}_{1}=\overline{\mathrm{n}}_{3}-\overline{\mathrm{n}}_{1}\right)$ war um $4.9 \mathrm{~cm} * * *$ pflanzenverlängend. 


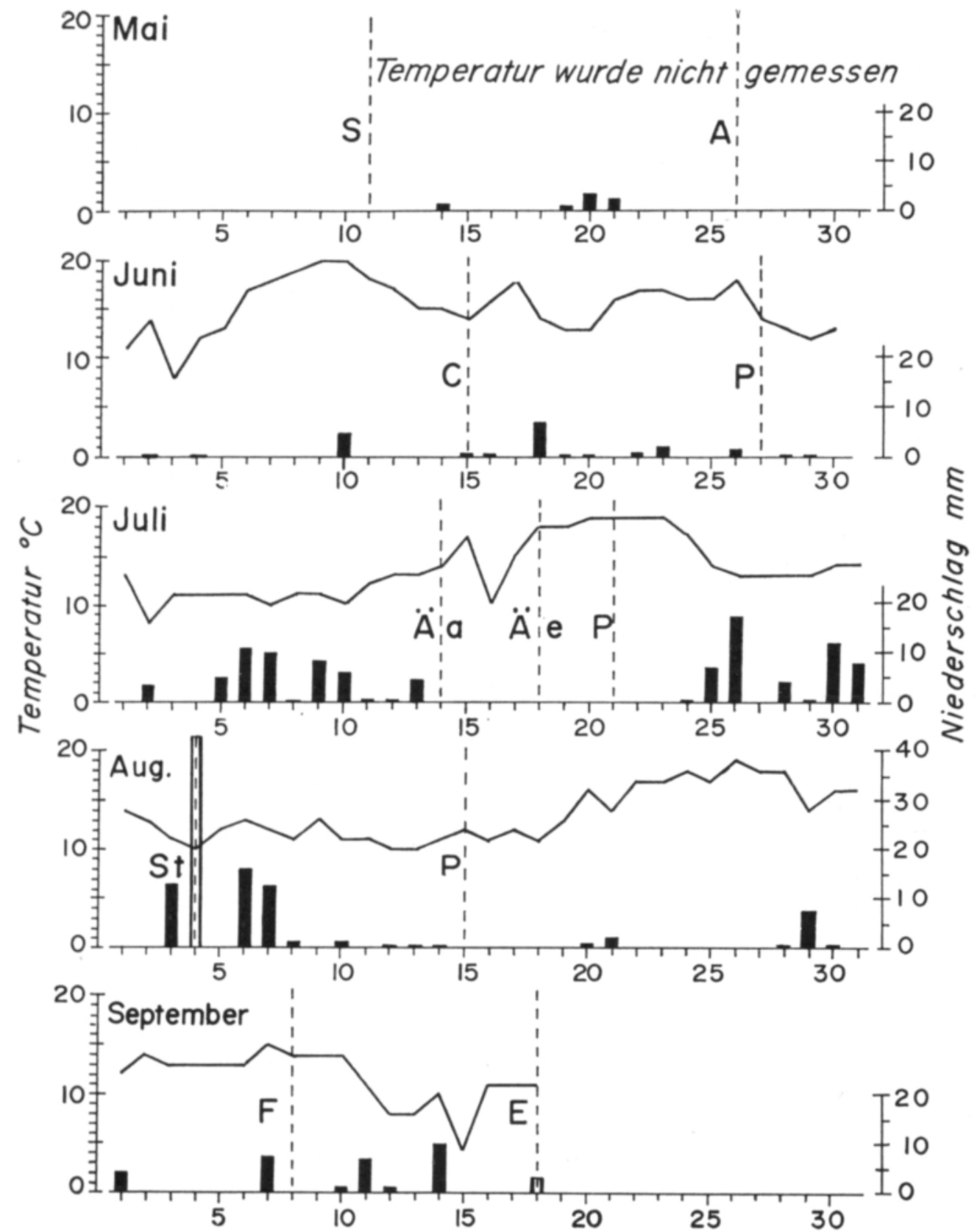

Abb. 1. Die Tagestemperaturen und -niederschläge samt wichtigen Zeitpunkten in der Entwicklung der Versuchspflanze.
$\mathrm{S}=$ Saat
A $=$ Aufspriessen
$\mathrm{C}=$ CCC-Spritzung
Äa $=$ Ährenschieben, Anfang
$\mathrm{A} \mathrm{e}=\quad$ Ende
$\mathrm{P}=$ Sammeln der Pflanzenproben
St $=$ Schätzen der Standfestigkeit
$\mathrm{F}=$ Bestimmung der Feuchtigkeit im Korn
$\mathrm{E}=$ Ernte


Die Halmlänge wurde von den am 15. 8. genommenen Pflanzenproben gemessen. Unten werden die Ergebnisse als Mittelwerte von 240 Messungen angeführt. Die Längen wurden von der Bodenoberfläche bis zum unteren Ende der Ähre in Zentimeter gemessen.

$\begin{array}{ccccc} & \mathrm{n}_{1} & \mathrm{n}_{2} & \mathrm{n}_{3} & \overline{\mathrm{n}} \\ \mathrm{c}_{0} & 65.2 & 68.0 & 72.6 & 68.6 \\ \mathrm{c}_{1} & 44.3 & 47.5 & 54.8 & 48.9 \\ \overline{\mathrm{c}} & 54.7 & 57.7 & 63.7 & 58.7\end{array}$

CCC veranlasste, dass der Halm durchschnittlich $19.7 \mathrm{~cm} * * *$ oder ca. $29 \%$ kürzer als bei den unbehandelten Pflanzen blieb. Die Stickstoffmenge von $100 \mathrm{~kg} / \mathrm{ha}$ verursachte das Wachsen der Halme $9.0 \mathrm{~cm} * * *$ länger als die Stickstoffmenge von $25 \mathrm{~kg} / \mathrm{ha}$.

In der Tabelle 1 werden die Längen der Internodien am 21. 7. und 15. 8. als Mittelwerte von 120 Messungen angeführt. Die Internodien sind von oben an numeriert: das 1. Internodium $=$ das oberste Internodium. Am 21. 7. wurden drei, am 15. 8. vier oberste Internodien gemessen. Dazu wird in der Tabelle noch das Wachsen der drei obersten Internodien in der Zeit vom 21. 7. bis zum 15. 8. angeführt.

Tabelle 1. Länge der Internodien $\mathrm{cm}$.

\begin{tabular}{|c|c|c|c|c|c|c|c|c|c|c|}
\hline \multicolumn{2}{|c|}{ Datum } & \multicolumn{2}{|c|}{ 21. 7 . } & \multicolumn{4}{|c|}{ 15. 8.} & \multicolumn{3}{|c|}{$\begin{array}{c}\text { Wachsen in der Zeit } \\
\text { 21. 7. }-15.8 .\end{array}$} \\
\hline Interno & ium 1. & 2. & 3. & 1. & 2. & 3. & 4. & 1. & 2. & 3. \\
\hline $\mathrm{n}_{1} \mathrm{c}_{0}$ & 27.3 & 15.8 & 8.7 & 37.1 & 17.4 & 9.2 & 4.4 & 9.8 & 1.6 & 0.5 \\
\hline$c_{1}$ & 20.6 & 9.4 & 5.2 & 26.1 & 10.4 & 5.4 & 3.0 & 5.5 & 1.0 & 0.2 \\
\hline $\mathrm{n}_{2} \mathrm{c}_{0}$ & 31.0 & 17.0 & 9.2 & 36.6 & 17.5 & 9.4 & 4.7 & 5.6 & 0.5 & 0.2 \\
\hline$c_{1}$ & 23.5 & 11.3 & 5.8 & 27.6 & 11.5 & 6.3 & 3.4 & 4.1 & 0.2 & 0.5 \\
\hline $\mathrm{n}_{3} \mathrm{c}_{0}$ & 31.2 & 18.4 & 9.5 & 38.9 & 18.8 & 10.3 & 4.4 & 7.7 & 0.4 & 0.7 \\
\hline$c_{1}$ & 25.2 & 14.4 & 7.4 & 29.6 & 13.5 & 7.4 & 3.4 & 4.4 & -0.9 & 0.0 \\
\hline$\overline{\mathrm{n}}_{1}$ & 23.9 & 12.6 & 6.9 & 31.6 & 13.9 & 7.3 & 3.7 & 7.7 & 1.3 & 0.4 \\
\hline$\overline{\mathrm{n}}_{2}$ & 27.2 & 14.1 & 7.5 & 32.1 & 14.5 & 7.8 & 4.0 & 4.9 & 0.4 & 0.3 \\
\hline$\overline{\mathrm{n}}_{3}$ & 28.2 & 16.4 & 8.4 & 34.2 & 16.1 & 8.8 & 3.9 & 6.0 & -0.3 & 0.4 \\
\hline $\bar{c}_{0}$ & 29.8 & 17.1 & 9.1 & 37.5 & 17.9 & 9.6 & 4.5 & 7.7 & 0.8 & 0.5 \\
\hline $\bar{c}_{1}$ & 23.1 & 11.7 & 6.1 & 27.8 & 11.8 & 6.3 & 3.2 & 4.7 & 0.1 & 0.2 \\
\hline $\bar{x}$ & 26.4 & 14.4 & 7.6 & 32.6 & 14.8 & 8.0 & 3.8 & 6.2 & 0.4 & 0.4 \\
\hline $\mathrm{N}_{1}$ & $+4.3^{*}$ & $+3.8^{* * * *}$ & $+1.5^{* * *}$ & $+2.6^{* *}$ & $+2.2^{* *}$ & $+1.5^{* * *}$ & +0.2 & -1.7 & $-1.6^{* *}$ & 0.0 \\
\hline $\mathrm{C}$ & $-6.7 * * *$ & $-5.4 * * *$ & $-3.0 * * *$ & $-9.7 * * *$ & $-6.1^{* * *}$ & $-3.3^{* * *}$ & $-1.3^{* * *}$ & $-3.0^{*}$ & -0.7 & -0.3 \\
\hline $\mathrm{N}_{1} \mathrm{xC}$ & +0.4 & $+1.2^{* *}$ & $+0.7 * *$ & +0.9 & +0.8 & +0.5 & +0.2 & +0.5 & -0.4 & -0.2 \\
\hline
\end{tabular}


Die CCC-Spritzung hatte die Wirkung, dass alle gemessenen Internodien kürzer als bei den unbehandelten Pflanzen blieben. Die CCC-Spritzung wirkte noch in der Zeit vom 21. 7. bis zum 15. 8., weil wenigstens das zweite Internodium der gespritzten Pflanzen weniger als das der ungespritzten wuchs.

Bei der verstärkten Stickstoffdüngung wurden die Internodien länger. Das Wachsen der Internodien in der Zeit vom 21. 7. bis 15. 8. scheint jedoch grösser auf den Parzellen gewesen zu sein, die wenig (25 kg N/ha) als auf den, die viel Stickstoff (100 kg/ha) bekommen hatten.

Die Wechselwirkung zwischen der linearen Komponente der Stickstoffdüngung und der CCC-Spritzung $\left(\mathrm{N}_{1} \mathrm{xC}=\overline{\mathrm{n}_{3} \mathrm{c}_{1}}-\overline{\mathrm{n}_{3} \mathrm{c}_{0}}-\overline{\mathrm{n}_{1} \mathrm{c}_{1}}+\overline{\mathrm{n}_{1} \mathrm{c}_{0}}\right)$ war am 21. 7. bei dem 2 . und 3. Internodium deutlich positiv. Eine hohe Stickstoffdüngung hatte also den Einfluss der CCC-Spritzung auf das Längenwachstum der Internodien bis zum 21. 7. vermindert. Am 15. 8. waren keine deutlichen Unterschiede mehr vorhanden.

Indem die Internodien und somit der ganze Halm kürzer blieben, hatte dies die Erhöhung der Standfestigkeit zur Folge, was aus den am 4. 8. nach einem Tagesniederschlag von $43 \mathrm{~mm}$ geschätzten Standfestigkeiten (Skala: $0=$ ganz am Boden liegend, $10=$ aufrecht) hervorgeht:

$\begin{array}{rrrrr} & \mathrm{n}_{1} & \mathrm{n}_{2} & \mathrm{n}_{3} & \overline{\mathrm{n}} \\ & & & & \\ \mathrm{c}_{0} & 7.5 & 7.9 & 5.8 & 7.0 \\ \mathrm{c}_{1} & 10.0 & 9.7 & 9.3 & 9.7 \\ \overline{\mathrm{c}} & 8.7 & 8.8 & 7.5 & 8.3\end{array}$

Das CCC erhöhte also die Standfestigkeit (2.7*** Einheiten) merkbar mehr als sie durch die Steigerung der Stickstoffdüngung von 25 auf $100 \mathrm{~kg} / \mathrm{ha}$ verringert wurde (1.2*** Einheiten).

Nach Tolbert (1960) verursacht das CCC eine Verzögerung der generativen Entwicklung der Pflanze. Das Ährenschieben verspätete sich in der Tat auf den CCCbehandelten Parzellen 2-3 Tage im Vergleich mit den unbehandelten. Dagegen scheint das Reifen auf den behandelten Parzellen früher als auf den unbehandelten stattgefunden zu haben, was aus den 10 Tage vor der Ernte bestimmten Feuchtigkeiten (\%) des Kornes hervorgeht:

$\begin{array}{ccccc} & \mathrm{n}_{1} & \mathrm{n}_{2} & \mathrm{n}_{3} & \overline{\mathrm{n}} \\ \mathrm{c}_{0} & 31.1 & 32.5 & 32.3 & 31.9 \\ \mathrm{c}_{1} & 28.2 & 28.4 & 30.4 & 29.0 \\ \overline{\mathrm{c}} & 29.6 & 30.4 & 31.3 & 30.5\end{array}$

Das aus den mit CCC gespritzten Pflanzen gedroschene Korn war 2.9***\% \%Einheiten trockener als das aus den ungespritzten Pflanzen, was ein Beweis für eine beschleunigende Wirkung der CCC-Spritzung auf das Reifen des Kornes ist. In diesem Versuch verursachte eine hohe Stickstoffdüngung $(100 \mathrm{~kg} \mathrm{~N} / \mathrm{ha})$ im Vergleich mit einer spärlichen $(25 \mathrm{~kg} \mathrm{~N} / \mathrm{ha})$, dass das Korn um $1.7 * * 0 \%$-Einheiten feuchter blieb. Die Steigerung der Stickstoffdüngung verzögerte das Reifen jedoch weniger als es von der CCC-Spritzung beschleunigt wurde. 
Bis zur Ernte (am 18. 9.), wo die Feuchtigkeit des Kornes nur noch $22.4 \%$ im Durchschnitt war, hatten sich die durch die Behandlungen verursachten Unterschiede merkbar ausgeglichen $\left(\mathrm{N}_{1}=+0.7 \%\right.$-Einh.*, $\mathrm{C}=-0.8 \%$-Einh.***).

Ertrag s e rgeb n is se. In der Tabelle 2 werden neben den Korn- und Stroherträgen die Pflanzenmassen am 27. 6. angeführt. Dazu werden in der Tabelle noch die durchschnittliche Grösse des Kornes und die Kornzahl der Flächeneinheit vorgebracht.

Tabelle 2. Evtragsergebnisse.

\begin{tabular}{|c|c|c|c|c|c|}
\hline & $\begin{array}{c}\text { Pflanzenmasse } 27.6 . \\
\text { kg Tr.S./ha }\end{array}$ & $\begin{array}{l}\text { Kornertrag } \\
\text { kg Tr.S./ha }\end{array}$ & $\begin{array}{c}\text { Korngrösse } \\
\mathrm{mg} / \mathrm{St} \text {. }\end{array}$ & $\begin{array}{l}\text { Kornzahl } \\
\text { St./m² }\end{array}$ & $\begin{array}{l}\text { Strohertrag } \\
\text { kg Tr.S./ha }\end{array}$ \\
\hline $\mathrm{n}_{1} \mathrm{c}_{0}$ & 924 & 1697 & 22.1 & 7700 & 1448 \\
\hline$c_{1}$ & 941 & 1455 & 19.2 & 7600 & 1091 \\
\hline $\mathrm{n}_{2} \mathrm{c}_{0}$ & 1160 & 1922 & 21.4 & 9000 & 1643 \\
\hline$c_{1}$ & 1080 & 1678 & 19.1 & 8800 & 1306 \\
\hline $\mathrm{n}_{3} \mathrm{c}_{0}$ & 1330 & 2129 & 20.5 & 10370 & 2053 \\
\hline$c_{1}$ & 1277 & 1856 & 18.5 & 10010 & 1600 \\
\hline$\overline{\mathrm{n}}_{1}$ & 932 & 1576 & 20.6 & 7650 & 1268 \\
\hline $\bar{n}_{2}$ & 1120 & 1800 & 20.2 & 8900 & 1474 \\
\hline$\overline{\mathrm{n}}_{3}$ & 1303 & 1992 & 19.5 & 10190 & 1826 \\
\hline $\bar{c}_{0}$ & 1138 & 1916 & 21.3 & 9020 & 1714 \\
\hline $\bar{c}_{1}$ & 1099 & 1662 & 18.9 & 8800 & 1332 \\
\hline $\bar{x}$ & 1118 & 1788 & 20.1 & 8910 & 1552 \\
\hline $\mathrm{N}_{1}$ & $+371^{* * *}$ & $+416^{* * * *}$ & $-1.1 * * *$ & $+2540^{* * *}$ & $+558^{* * *}$ \\
\hline $\mathrm{C}$ & -39 & $-254^{* * *}$ & $-2.4^{* * *}$ & -220 & $-382^{* * *}$ \\
\hline
\end{tabular}

Die CCC-Spritzung verminderte den Kornertrag in diesem Versuch um $13.3 \%$ $(254 \mathrm{~kg} / \mathrm{ha})$ und den Strohertrag um $22.3 \%$ (382 kg/ha). Am 27. 6. war die Wirkung des CCC noch nicht wahrnehmbar. Die ertragvermindernde Wirkung des CCC beruhte nur auf der Verkleinerung der Korngrösse, die Kornzahl der Flächeneinheit wurde nicht verändert.

Die positive Wirkung der verstärkten Stickstoffdüngung war schon am 27.6. sichtlich. Bei der Erhöhung der Stickstoffdüngung erhöhte sich der Kornertrag, indem sich die Kornzahl der Flächeneinheit vermehrte, trotz der gleichzeitigen Verminderung der Korngrösse. Auch der Strohertrag war auf den mit Stickstoff am meisten gedüngten Parzellen der grösste.

Über die chemische Zusammensetzung der Pflanze. In der Tabelle 3 werden die Gehalte an dem durch den gewöhnlichen Kjeldahl-Auf- 
Tabelle 3. Nährstoffgehalt in Prozent von Trockensubstanz.

\begin{tabular}{|c|c|c|c|c|c|c|c|c|c|}
\hline & \multicolumn{3}{|c|}{ In der Pflanzenmasse 27. 6 . } & \multicolumn{3}{|c|}{ Im Korn } & \multicolumn{3}{|c|}{ Im Stroh } \\
\hline & $\mathrm{N}$ & $\mathbf{P}$ & $\mathrm{K}$ & $\mathrm{N}$ & $\mathrm{P}$ & K & $\mathrm{N}$ & $\mathbf{P}$ & $\mathrm{K}$ \\
\hline $\mathrm{n}_{1} \mathrm{c}_{0}$ & 3.25 & 0.47 & 4.02 & 2.21 & 0.47 & 0.59 & 0.78 & 0.12 & 0.76 \\
\hline$c_{1}$ & 3.35 & 0.49 & 4.02 & 2.33 & 0.48 & 0.64 & 1.02 & 0.18 & 0.91 \\
\hline $\mathrm{n}_{2} \mathrm{c}_{0}$ & 3.70 & 0.47 & 4.36 & 2.25 & 0.46 & 0.58 & 0.78 & 0.13 & 0.81 \\
\hline$c_{1}$ & 3.77 & 0.48 & 4.14 & 2.36 & 0.49 & 0.63 & 1.01 & 0.18 & 0.98 \\
\hline $\mathrm{n}_{3} \mathrm{c}_{0}$ & 4.06 & 0.46 & 4.19 & 2.43 & 0.47 & 0.60 & 1.02 & 0.14 & 1.03 \\
\hline$c_{1}$ & 4.31 & 0.48 & 4.28 & 2.56 & 0.50 & 0.64 & 1.13 & 0.17 & 1.20 \\
\hline$\overline{\mathrm{n}}_{1}$ & 3.30 & 0.48 & 4.02 & 2.27 & 0.47 & 0.61 & 0.89 & 0.15 & 0.83 \\
\hline$\overline{\mathrm{n}}_{2}$ & 3.73 & 0.48 & 4.25 & 2.30 & 0.47 & 0.60 & 0.89 & 0.15 & 0.90 \\
\hline$\overline{\mathrm{n}}_{3}$ & 4.18 & 0.47 & 4.23 & 2.50 & 0.48 & 0.62 & 1.07 & 0.15 & 1.11 \\
\hline$\overline{\mathrm{c}}_{0}$ & 3.67 & 0.47 & 4.18 & 2.30 & 0.47 & 0.59 & 0.85 & 0.13 & 0.86 \\
\hline $\bar{c}_{1}$ & 3.81 & 0.49 & 4.14 & 2.42 & 0.49 & 0.63 & 1.05 & 0.17 & 1.03 \\
\hline $\bar{x}$ & 3.74 & 0.48 & 4.16 & 2.36 & 0.48 & 0.61 & 0.95 & 0.15 & 0.95 \\
\hline $\mathrm{N}_{1}$ & $+0.88^{* * *}$ & -0.01 & $+0.21^{* * *}$ & $+0.23^{* * *}$ & +0.01 & +0.01 & $+0.18^{* * *}$ & 0.00 & $+0.28^{* * *}$ \\
\hline $\mathrm{N}_{\mathrm{q}}$ & -0.01 & +0.005 & $+0.13^{*}$ & $-0.09 * * *$ & -0.005 & -0.015 & $-0.09^{* * *}$ & 0.00 & $-0.07 * *$ \\
\hline C & $+0.14^{*}$ & +0.02 & -0.04 & $+0.12^{* * *}$ & $+0.02^{* * *}$ & $+0.04 * * *$ & $+0.20^{* * *}$ & $+0.04 * * *$ & $+0.17 * * *$ \\
\hline
\end{tabular}

schluss bestimmten Gesamt-Stickstoff und an den von einer Aschenlösung bestimmten Gesamt-Phosphor und -Kalium angeführt.

CCC erhöhte den Gehalt an Gesamt-Stickstoff im Pflanzenmaterial schon zwölf Tage nach der Spritzung (27.6.). Die Gehalte an den bestimmten Nährstoffen im Korn und besonders im Stroh waren wegen der CCC-Behandlung merkbar höher.

Eine höhere Stickstoffdüngung verursachte einen höheren Stickstoffgehalt in allen Proben. Bei der Ernte verursachte allerdings erst die Steigerung der Stickstoffdüngung von 50 auf $100 \mathrm{~kg} /$ ha eine Erhöhung des Stickstoffgehaltes in der Pflanze (im Korn und Stroh). Erwähnt sei in diesem Zusammenhang, dass die Abweichung der Wirkung von Stickstoff von der linearen durch die quadrantische Komponente $\left(\mathrm{N}_{\mathrm{q}}=\overline{\mathrm{n}}_{2}-\frac{\overline{\mathrm{n}}_{1}+\overline{\mathrm{n}}_{3}}{2}\right)$ ausgedrückt wird. Auf den Gehalt an Gesamt-Phosphor in der Pflanze hatte die Stickstoffdüngung keinen Einfluss, dagegen war der Gehalt an Gesamt-Kalium in der jungen Pflanzenmasse (27. 6.) und im Stroh wesentlich höher bei der Stickstoffdüngungsstufe $100 \mathrm{~kg} \mathrm{~N} / \mathrm{ha}$ als bei $25 \mathrm{~kg} \mathrm{~N} / \mathrm{ha}$. Die Steigerung der Stickstoffdüngung von 50 auf $100 \mathrm{~kg} /$ ha konnte allerdings nicht mehr den Gehalt an Kalium in dem jungen Pflanzenmaterial (27. 6.) erhöhen, dagegen erhöhte sich der Kaliumgehalt im Stroh noch bei der genannten Stickstoffdüngungsstufe merkbar. Die Tabelle 4 drückt die Nährstoffaufnahme der Pflanze aus. Darin werden die 
Tabelle 4. Die Nährstoffmengen in $\mathrm{kg} / \mathrm{ha}$ in derPflanzenmasse am 27. 6. und bei der Ernte.

\begin{tabular}{|c|c|c|c|c|c|c|c|c|c|}
\hline & \multicolumn{3}{|c|}{ Stickstoff } & \multicolumn{3}{|c|}{ Phosphor } & \multicolumn{3}{|c|}{ Kalium } \\
\hline & 27. 6 . & Korn & Stroh & 27. 6 . & Korn & Stroh & 27. 6 . & Korn & Stroh \\
\hline $\mathrm{n}_{1} \mathrm{c}_{0}$ & 29.8 & 37.6 & 11.3 & 4.37 & 7.94 & 1.76 & 37.1 & 9.9 & 11.0 \\
\hline $\mathrm{c}_{1}$ & 31.6 & 34.0 & 11.1 & 4.61 & 6.94 & 1.86 & 37.8 & 9.2 & 9.9 \\
\hline $\mathrm{n}_{2} \mathrm{c}_{0}$ & 42.7 & 43.3 & 12.8 & 5.47 & 8.79 & 2.01 & 50.9 & 11.1 & 13.4 \\
\hline$c_{1}$ & 40.7 & 39.6 & 13.2 & 5.20 & 8.11 & 2.22 & 44.8 & 10.6 & 13.0 \\
\hline $\mathrm{n}_{3} \mathrm{c}_{0}$ & 53.8 & 51.8 & 20.8 & 6.06 & 9.92 & 2.72 & 55.7 & 12.8 & 21.1 \\
\hline$c_{1}$ & 55.1 & 47.6 & 18.0 & 6.10 & 9.14 & 2.67 & 54.8 & 11.8 & 19.3 \\
\hline$\overline{\mathrm{n}}_{1}$ & 30.7 & 35.8 & 11.2 & 4.49 & 7.44 & 1.81 & 37.4 & 9.6 & 10.4 \\
\hline$\overline{\mathrm{n}}_{2}$ & 41.7 & 41.4 & 13.0 & 5.33 & 8.44 & 2.12 & 47.7 & 10.8 & 13.1 \\
\hline$\overline{\mathrm{n}}_{3}$ & 54.4 & 49.6 & 19.4 & 6.07 & 9.52 & 2.69 & 55.2 & 12.3 & 20.1 \\
\hline $\bar{c}_{0}$ & 42.1 & 44.2 & 14.9 & 5.30 & 8.88 & 2.16 & 47.8 & 11.2 & 15.2 \\
\hline $\bar{c}_{1}$ & 42.4 & 40.4 & 14.1 & 5.30 & 8.06 & 2.25 & 45.7 & 10.5 & 14.0 \\
\hline $\bar{x}$ & 42.2 & 42.2 & 14.5 & 5.30 & 8.46 & 2.21 & 46.7 & 10.9 & 14.6 \\
\hline $\mathrm{N}_{1}$ & $+23.7^{* * *}$ & $+13.8^{* * *}$ & $+8.2^{* * *}$ & $+1.58^{* *}$ & $+2.08^{* * *}$ & $+0.88^{* * *}$ & $+17.8^{* * *}$ & $+2.7 * * *$ & $+9.7 * * *$ \\
\hline $\mathrm{N}_{\mathrm{q}}$ & -0.9 & -1.3 & $-2.3^{* *}$ & +0.05 & -0.04 & -0.13 & +1.4 & -0.2 & -2.2 \\
\hline $\mathrm{C}$ & +0.3 & $-3.8^{* * *}$ & -0.8 & 0.00 & $-0.82^{* * *}$ & +0.09 & -2.1 & $-0.7 * * *$ & -1.2 \\
\hline
\end{tabular}

in der jungen Pflanzenmasse (27.6.), im Korn und im Stroh enthaltenen Nährstoffmengen (Stickstoff, Phosphor und Kalium) angeführt.

Das CCC verursachte in diesem Versuch eine deutliche Verminderung der Nährstoffmengen im Korn. Dagegen veranlasste das CCC keine statistisch signifikanten Unterschiede in den in der jungen Pflanzenmasse und im Stroh enthaltenen Nährstoffmengen.

Die Vermehrung der Stickstoffdüngung hatte eine verbesserte Aufnahme aller Nährstoffe zur Folge.

\section{Diskussion}

Das auf $20 \mathrm{~cm}$ hohe Sommerweizenpflanzen gespritzte CCC $(3 \mathrm{~kg} / \mathrm{ha})$ erhöhte die Standfestigkeit des Getreides mehr, als sie durch die Steigerung der Stickstoffdüngung von 25 auf $100 \mathrm{~kg} /$ ha vermindert wurde. Die Wirkung der CCC-Spritzung beruhte wahrscheinlich darauf, dass der Halm dadurch ca. $20 \mathrm{~cm}$ kürzer blieb, was die Folge der Verkürzung aller Internodien war.

Neben dem kürzeren Halm verursachte das CCC eine Verringerung des Strohertrages. Auch auf den Kornertrag hatte das CCC eine vermindernde Wirkung, die allerdings durch die gleichzeitige Steigerung der Stickstoffdüngung von 25 auf 50 
oder von 50 auf $100 \mathrm{~kg} / \mathrm{ha}$ ungefähr kompensiert wurde. Durch die Steigerung der Stickstoffdüngung von 25 auf $100 \mathrm{~kg} / \mathrm{ha}$ neben der Spritzung mit CCC wurde der Kornertrag um $160 \mathrm{~kg} /$ ha vergrössert, während auch die Standfestigkeit zunahm.

Der Minderertrag durch das CCC beruhte wahrscheinlich darauf, dass das Wachsen des Kornes unterbrochen wurde, was daraus zu schliessen ist, dass die mit CCC gespritzten Parzellen früher als die übrigen reif wurden. Man konnte auch feststellen, dass das Korn der mit CCC behandelten Pflanzen kleiner als das der unbehandelten blieb. Dagegen wurden bei der Zahl der Körner der Flächeneinheit keine durch das CCC veranlassten Unterschiede bemerkt, während der Mehrertrag durch die Steigerung der Stickstoffdüngung einer merkbaren Zunahme der Kornzahl zuzuschreiben war.

Mit der vermehrten Stickstoffdüngung nahm die Aufnahme aller untersuchten Pflanzennährstoffe (N, P und K) zu, worauf die festgestellten Mehrerträge wahrscheinlich beruhten. Das CCC verursachte keinen deutlichen Unterschied in der Nährstoffaufnahme der Pflanze bis zur Ernte, obgleich die Nährstoffmengen im Korn abnahmen. Dagegen nahmen die Gehalte an den Nährstoffen im Korn und im Stroh wegen der Verminderung der entsprechenden Erträge zu. Der neben dem Stickstoffgehalt offenbar gestiegene Gehalt an Proteinen verbesserte möglicherweise die Qualität des Kornes.

\section{Zus a m m e nfass $u$ g}

Im Jahre 1965 wurde in Hausjärvi $\left(60^{\circ} 46^{\prime}\right.$ nördl. Breite, $24^{\circ} 51^{\prime}$ östl. Länge) auf einem lehmigen Sandboden ein Feldversuch zu dem Zweck durchgeführt, die Möglichkeiten des auf junge Pflanzen gespritzten Chlorcholinchlorids (CCC) für die Erhöhung der Standfestigkeit von der Sommerweizensorte Norröna zu ermitteln. Um das Lagern hervorzurufen, wurde Stickstoffdüngung in drei Stufen (25, 50 und $100 \mathrm{~kg} \mathrm{~N} / \mathrm{ha}$ ) gegeben.

Die Spritzung mit CCC (3 kg/ha) verursachte eine deutliche Erhöhung der Standfestigkeit und zwar aus dem Grunde, dass alle Internodien kürzer und somit der gesamte Pflanzenbestand niedriger blieben. Zugleich verminderte sich allerdings der Kornertrag um $13 \%$. Dies beruhte wahrscheinlich darauf, dass das Wachsen des Kornes vorzeitig unterbrochen wurde. Der Minderertrag konnte verhindert werden, indem die gleichzeitig mit der CCC-Spritzung zu gebende Stickstoffdüngung gesteigert wurde. Zugleich konnte die Standfestigkeit noch erhöht werden.

Die CCC-Spritzung hatte keinen Einfluss auf die Nährstoffaufnahme der Pflanze, aber die Gehalte an den untersuchten Nährstoffen (N, P und K) nahmen als Folge der Spritzung mit CCC wegen der verminderten Korn- und Stroherträge zu.

\section{LITERATUR}

Linser, H. \& MAYR, H. \& Bodo, G. 1961. Uber die Wirkung von Chlorcholinchlorid auf Sommerweizen. Bodenkultur 12: 279-280.

MAYR, H. \& Primost, E. \& Rittmeyer, G. 1962. Untersuchungen über die Erhöhung der Standfestigkeit von Getreide. I. Feldversuche mit Chlorcholinchlorid zu Winterweizen. Ibid. 13: 27-45. 
Mukula, J. \& Teittinen, P. \& LaAksonheimo, J. 1965. Chlorocholine chloride (CCC) for prevention of lodging of wheat in Finland. Acta Agr. Fenn. 107: 103-124.

PRIMost, E. 1964. Die Wirkung von Chlorcholinchlorid (CCC) auf die Korn- und Stroherträge von Winterweizen in zwei witterungsmässig extremen Jahren. Z. Pflanzen- u. Ackerbau 119: 211 - 226.

Sturm, H. \& Jung, J. 1964. Der Einfluss von Blattspritzungen mit Chlorcholinchlorid (CCC) auf Wachstum und Ertrag von Weizen. Ibid. 120: 232-252.

TOLBERT, N. E. 1960. (2-chloroethyl)trimethylammonium chloride and related compounds as plant growth substances. II. Effect on growth of wheat. Plant Physiol. 35: 380-385.

\title{
SELOSTUS:
}

\section{KLOORKOLINKLORIDIN (CCC) VAIKUTUKSISTA KEVÄTVEHNÄN KASVUUN, SATOON JA RAVINTEIDEN OTTOON KENTTÄKOKEEN TULOSTEN PERUSTEELLA}

\author{
Antti JаAкKоLA
}

\section{Yliopiston maanviljelyskemian laitos, Helsinki}

V. 1965 suoritettiin Hausjärvellä hiesuisella karkealla hietamaalla kenttäkoe, jossa pyrittiin selvittämään oraille ruiskutetun $\mathrm{CCC}: \mathrm{n}$ mahdollisuuksia Norröna-kevätvehnän laontorjunnassa. Lakoutumisen aikaansaamiseksi kokeeseen kuului koetekijänå typpilannoitus kolmena asteena $(25,50$ ja $100 \mathrm{~kg} \mathrm{~N} / \mathrm{ha})$.

CCC-ruiskutus (3 kg CCC/ha) aiheutti lakoisuuden selvän vähenemisen, mikä johtui kaikkien nivelvälien lyhetessä madaltuneesta kasvustosta. Samalla jyväsato kuitenkin aleni n. $13 \%$. Tämăn arveltiin johtuneen jyvien kasvun ennenaikaisesta pysähtymisestä. Typpilannoitusta samanaikaisesti kohottamalla pystyttiin satotappio ehkäisemään, siten että laonkestävyys silti vielä parani.

CCC-ruiskutus ei vaikuttanut kasvin ravinteiden ottoon, mutta tutkittujen ravinteiden (typen, fosforin ja kaliumin) pitoisuudet lisääntyivät CCC-ruiskutuksen seurauksena sekä jyvissä että oljissa vähentyneiden jyvä- ja olkisatojen takia. 